\title{
Hybrid Loop Controlled Dual Axis Mechanical Solar Tracking Systems and MPPT for Nano/Micro Grid Applications
}

\author{
Gorijala Anitha, N. Prema Kumar
}

\begin{abstract}
This paper emphasizes the important role of solar tracker systems in Micro/Nano grid level applications. Basically mechanical and electrical type trackers are different in operation but they are similar in way of tracking of solar energy. Mechanical trackers (Dual axis solar tracking system) are widely used where space is less and quality is more and with fixed panels solar MPPT is adoptable. Hybrid loop control which controls the relative motion of solar tracking system (STS) during the day as per the position of sun. Hybrid loop control (HLC) is effective rather than open and closed loop systems due its mode of control using sun position algorithm and sensors based as per the requirement and environmental conditions. Most commonly used sensor based trackers takes feedback to adjust the panel position in such a way to track the sun, but the situation when the sky is cloudy creates problem in performance of the system. The estimated accuracy is very close to $\pm 0.0003^{\circ}$ but it depends on the irradiation coming from sun. So, Sun tracking system by using solar position algorithm with accuracy is required in mechanical solar trackers and maximum power point tracking in electrical trackers. In order improve the efficiency of whole solar tracking system, we incorporated both open and closed loops i.e., hybrid loop control which plays important role to improve the overall performance. In view of that we incorporated both open and closed loops i.e., hybrid loop control. Here we presented Maximum power point tracking(MPPT) which is electrical tracking system simulated in MATLAB environment and also presented mechanical type of tracking system It is facilitated by using Programmable logic controller $(P L C)$ to access more input/output controls and simulation has been done in CODESYS software using ladder logic programming.
\end{abstract}

Keywords :HLC, PLC, CODESYS software, MATLAB software, MPPT, STS, DASTS (Dual axis mechanical solar tracking system).

\section{INTRODUCTION}

Importance of Solar tracking system: Due to accessibility and low cost, clean energy resources based applications trending now. The solar system efficiency can be improved by increasing solar cell efficiency, MPPT (electrical solar tracking system) and mechanical solar tracking system [1] [7][13][15]. In particular a solar tracking system mechanism aligned in such a way that to increase efficiency of power from solar array. The conventional solar modules are fixed hence energy extracted from sun rays by the panel depends on various angles at various time especially the duration of sun rays which are perpendicular to the panel surface is very

Revised Manuscript Received on September 22, 2019.

* Correspondence Author

Aravelli.S.L.K.Gopalamma, Research Scholar, Dept. of EE, AUCE (A),Andhra University, Visakhapatnam, India

Dr.R.SrinuNaik, Assistant professor, Dept. of EE, AUCE (A), Andhra University, Visakhapatnam, India less which make the production of energy from panels is comparatively low. In order to overcome this problem Solar tracking mechanism is the best solution [1] [9][10].By the use of Solar tracking systems we can rotate the solar panels according to the rotation of sun across the sky. So, the energy output improves, thereby increasing its efficiency. In a day, sun position is dynamic in nature it changes rapidly in the sky, so tracking systems are advantageous to orient the collection system to make the system most effective. Zhang and group (2012) explains the calculated results and the performance of HSAT( horizontal single axis solar tracking) that which increased the efficiency up to $36 \%$ drastically [5].Yusie and group clearlypresented one of the method for sun tracking system by using solar position algorithm (SPA) with improved accuracy, Most commonly used sensor based trackers takes feedback to adjust the panel position in such a way to track the sun, but the situation when the sky is cloudy creates problem in performance of the system. The estimated accuracy is very close to $\pm 0.0003^{\circ}$ but it depends on the irradiation coming from sun[2].Concentrated solar power require accurate, precise control at focal point of the reflector. In case of Non- concentrated type of solar system needn't go for tracking, but if we did so tracking system can definitely Improves efficiency and these types of systems are very accurate and effective. In order to achieve the tracking mechanism gear mechanism is used to rotate the panel with respect to the speed of sun movement. Duration of sunrise and sun set is 12 hours and sun covers angle of 180 degrees in 12 hrs. And returns manually or automatically using appropriate controllers like PLC, Micro controllers etc. Thus a way that always the panels face sun rays in perpendicular. Design of solar tracking system works on the principle of real time clock and gearing equipment that which depends on rotation of the solar concentrator with solar movement. Simply, Sun complete its half of the revolution (180 deg) within $12 \mathrm{hrs}$. So the sun rotation per hour is $15 \mathrm{deg}$ per hour. Solar tracking systems are the equipment used to place PV panels, reflectors and other optical devices towards the sun [3]. SuneethaRacharla and group classified the different solar trackers and the performance of each is explained [4]. So far, work done on electrical and mechanical trackers in this paper we introduced the simulation of mechanical solar tracker in industrial applications under micro grid level using codesys software.

\section{METHODOLOGY}

Section 1. In this section we explained the national and international contribution towards solar energy and tracking systems. 
Section 2. In this sectionwe dealt with brief idea about solar tracking systems and hybrid loop control in mechanical solar tracking system and the process of its execution in step by step.

Section 3. Simulation results of both MPPT (electrical tracking)in MATLAB platform and mechanical tracking system (DASTS) in CODESYS software has been executed.

Section 4. This section concludes the motivation and contribution of work.

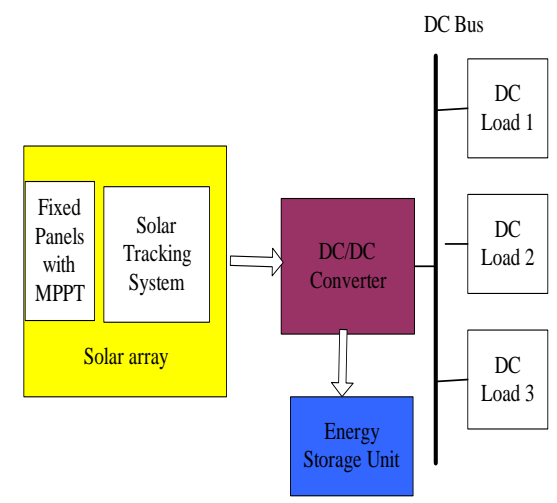

DC Nano Grid for Residential Applications/E-charging Station

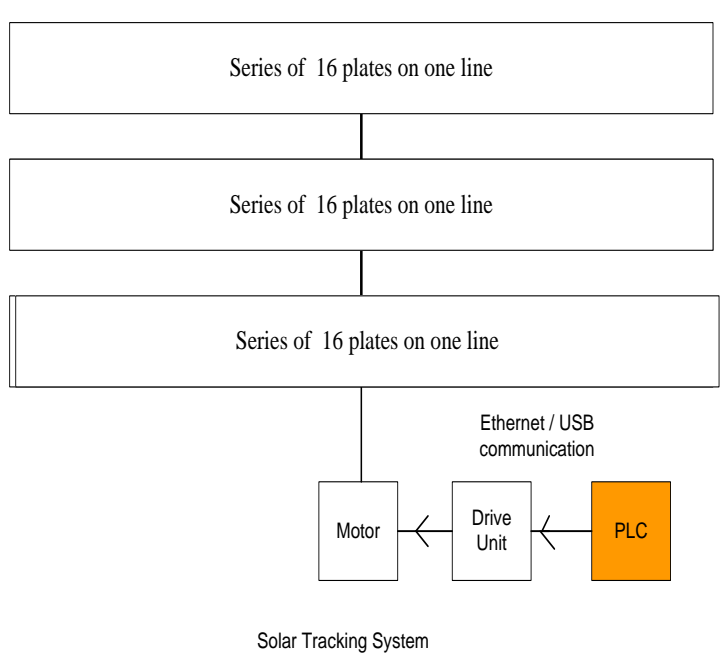

Figure 1: Schematic diagram of solar tracking system for micro/ Nano grid level

\section{SOLAR TRACKING SYSTEM COMPONENTS}

A. Types of mechanical solar Tracking System

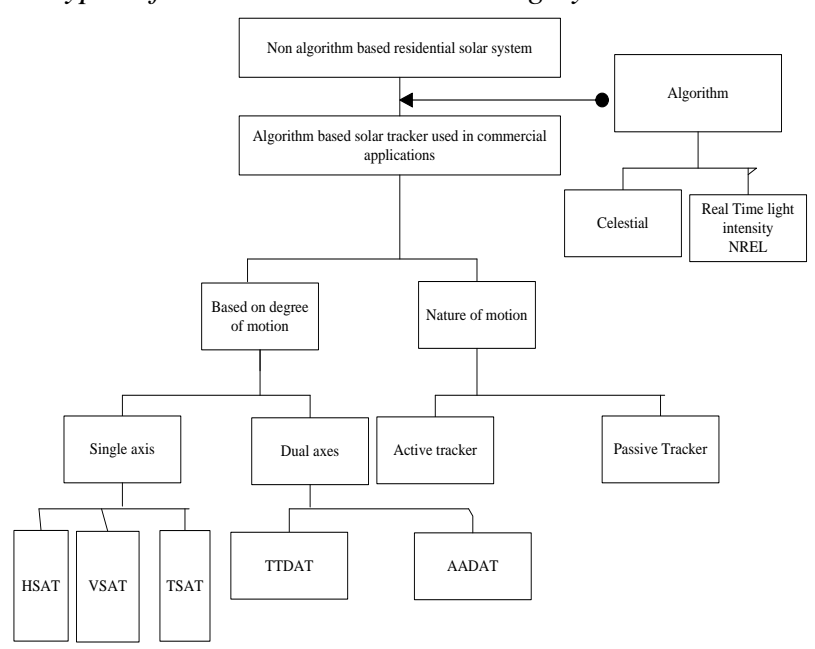

Figure:2 Types of Solar Tracking system

\section{B. Electrical solar Tracking systems:}

In present economic status of some nations have been focusing in reducing maintenance cost and effective cost. In regard the best way to track the solar energy is maximum power point tracking which is widely adapted. They are several methods involved out of all because of its simplicity perturb and observe method is worth to mention.[14][15]

1. Perturb and Observe (P\&O): In industries this algorithm is widely used. It works based on the comparison of generated power and respective duty cycle is generated.

2. Incremental Conductance: This algorithm is robust but complex in nature. The major Contribution of this method is comparison between instantaneous conductance and additional conductance and duty cycle adjusted accordingly.

3. Fractional Open Circuit Voltage: This algorithm works based on to maintain constant fraction of MPP voltage and open circuit voltage.

\section{Mechanical Solar system Components}

1. Sun tracking algorithm (Solar positioning algorithm): It calculates the azimuth and zenith angle of the sun and these angles are important to know the orientation and the position of the PV panel to face towards the sun in case of open loop/ closed loop systems or hybrid loop, But In case where wetake precision and accurate in operation, we employ methods to extract real time light intensity data and that has taken into account. In order to overcome the problem solar positioning algorithm is a solution. Parameters related to environmental conditions of the observer were taken from NREL (National Renewable Energy Laboratory) position algorithm calculator provides us the information about our region sun irradiation data. Geographical location data specification includespressure, elevation, time zone,temperature, longitude and latitude, surface azimuth rotation, and delta T,surface slope, once the local parameters dumped in the program then it calculates all the parameters as per the specified area such as Azimuth and zenith angles and sun rise, sun set for the duration of day calculation. The zenith angle is the angle between directions of sun to the directly overhead. UDFB (User defined function block) provides the output zenith angle as Out_Zenith. The azimuth angle is measured from north to the point on the horizontal directly below the project in clockwise. Out - Azimuthnavi output (0...360deg) denotes the navigator azimuth angle which is used in solar tracking applications [3].

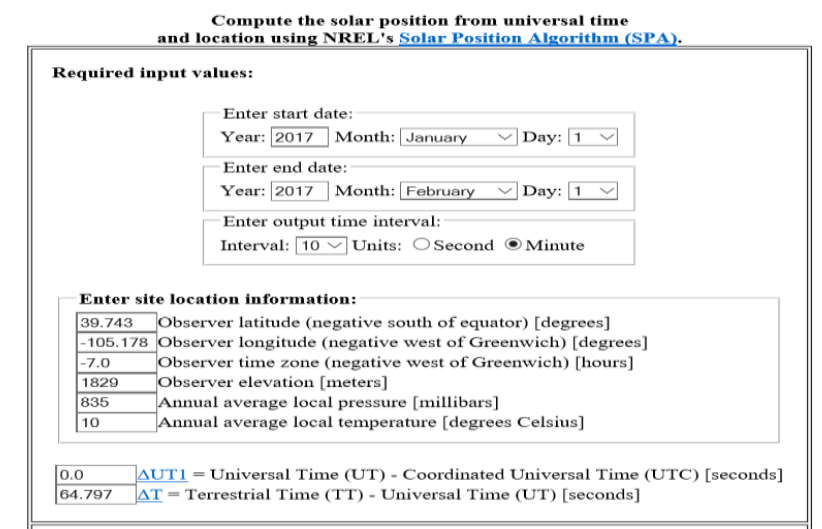

Figure 3: Data Extraction from NREL SPA

Published By:

Blue Eyes Intelligence Engineering \& Sciences Publication

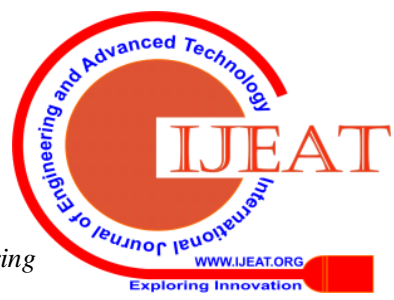


2. Control Unit: The control unit that which executes the sun tracking algorithm and also coordinates the rotation of the solar panels with precise and accurate. Micro Logix PLC: A Series of Micro Logix such as MICRO 810, MICRO 820, MICRO 830, MICRO 870, MICRO 800 Expansion I/O Modules, Micro800 PLC Plug-in Modules. Out of all Micro820 is Used In solar Tracking Systems which are used in solar power plants, Solar Parks, Commercial Residential areas. Variable frequency drive used to improve the efficiency by converting fixed $\mathrm{v}$ and $\mathrm{f}$ to variable $\mathrm{v}$ and $\mathrm{f}$.

3. Positioning system: positioning systems moves the system in such a way to face the sun based on azimuth angle, and these are electrical or hydraulic. In case of electrical systems one can utilize encoders and variable frequency drives / linear actuators to monitor the present position and to change if required.

Closed Loop control: Closed loop systems track the sun rays by using sensors in proper direction, directed at the sun. As the sun moves one or more sensors begin to shade such at it is fed back to inclinometer to measure the tilt angle and the same instruction will be given to activate the actuators/ motors to move the device to desired position. In such a way the outputs get maximized. Initialization parameters and control manually can ease with the implementation of Programmable touch screens. The PLC Ladder programming is relatively simple. But Controller system would facedifficultscenarioto recover from prolonged periods of cloud cover. In the absence of guidance from astronomical sun positioning algorithm it is a problem to determine the solar vector when sun moved out of sensor range.

Open loop control: Open loop systems basically work on mathematical formula to control device motors/ actuators to follow the sun movement. Real time clock is used in this type of control but accuracy is low compare to closed loop. [6][11] Hybrid loop control: By satisfying the roadblocks of both open loop and closed loop control hybrid loop control is preferable as it compares both position of sun from SPA and through sensors.

4. Drive Transmission: These include linear type actuators, drives, hydraulic cylinders, planetary gears, and threaded spindles. Power flex 525 an AC drive which offers wide range of power rating from $(0.5-30) \mathrm{HP}$ and they are ideal for applications such as conveys fans, pumps and mixers. Depending upon the location of the observer sun speed is $0.25 \mathrm{deg} / \mathrm{min}$ on average. Thus solar tracker has to achieve an angular rate of movement of at least $0.25 \mathrm{deg} / \mathrm{min}$ to keep the relative motion with the sun. Electric motors SQIM run at a speed $1500 \mathrm{rpm}$ range approximately. This means that the motor on its own would not achieve the minimum speed it is possible with Gear drive mechanism. Usually high gear ratio drive is preferred around 10000:1 or 30000: 1. [6]

5. Sensing Equipment: For the solar tracking system that which use light intensity real time data in the algorithm uses pyranometers to read the data. In order to optimize efficiency and production monitoring equipment for parameters such as pressure, temperature and humidity. In some cases Proximity sensor placed beneath of $\operatorname{cog}$ wheel above PLC which is used to sense the nearby object without contact. [13]

6. Limit Switches: are used in controlling speed and for mechanical over limits, it is very important to prevent damages involving in solar tracker system.
7. Elevation / positioning feedback: It is accomplished by using either combination of limit switches and encoder or inclinometer which is an instrument used for measuring angles of slope / tilt or elevation of panel with respect to the gravitational direction.

\section{HYBRID LOOP CONTROL}

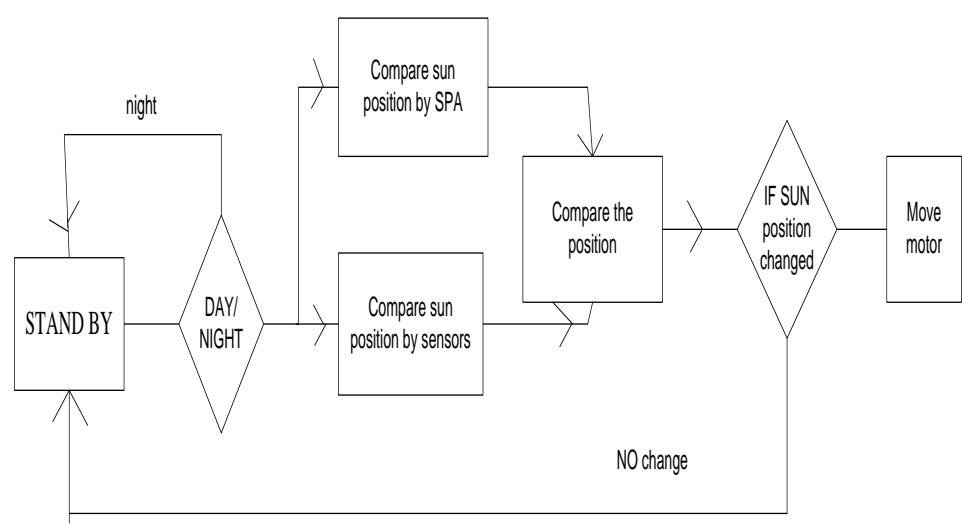

Figure 4: Hybrid loop control

PLC (Programmable logic Controller acts like a brain in human, it controls the movement of the DC motor and stepper motor directly as per the instructions given. Data received from the sensor arrangements which consist of LDRs and resistors are processed by the PLC. It controls the direction of DC motor in such a way to ensure the solar panel perpendicular towards the Sun. Output relays inside the PLC controls the rotation of the motor either to rotate clockwise or anticlockwise for horizontal as well as vertical axis. The solar panel that which is attached to the DC motor/ stepper motor will be controlled according to the direction of the dc motor and stepper motor whether to move in horizontal or vertical and it decides the direction to move in A or B .

\section{A. Light Dependent resistors:}

An LDR is a component that has a variable resistance that which changes with the variation of light intensity that falls upon it. This property allows them to be used in sensing circuits in closed loop solar Tracking System.

\section{B. Working Procedure of solar Tracking system}

Before Starting the process checking the connections as per specified data

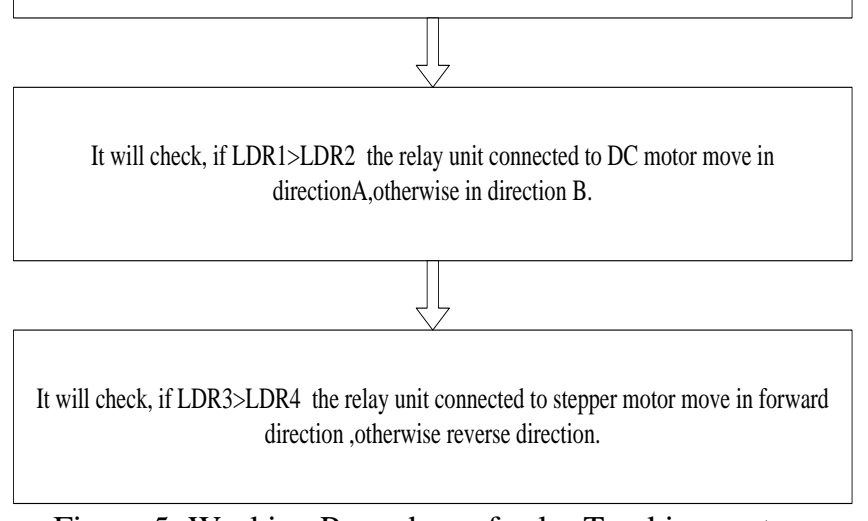

Figure 5: Working Procedure of solar Tracking system 
Hybrid loop controlled dual axis mechanical solar tracking systems and MPPTfor Nano/Micro grid Applications

\section{Simultion AND ReSUlts}

A. Electrical solar tracking system simulation:

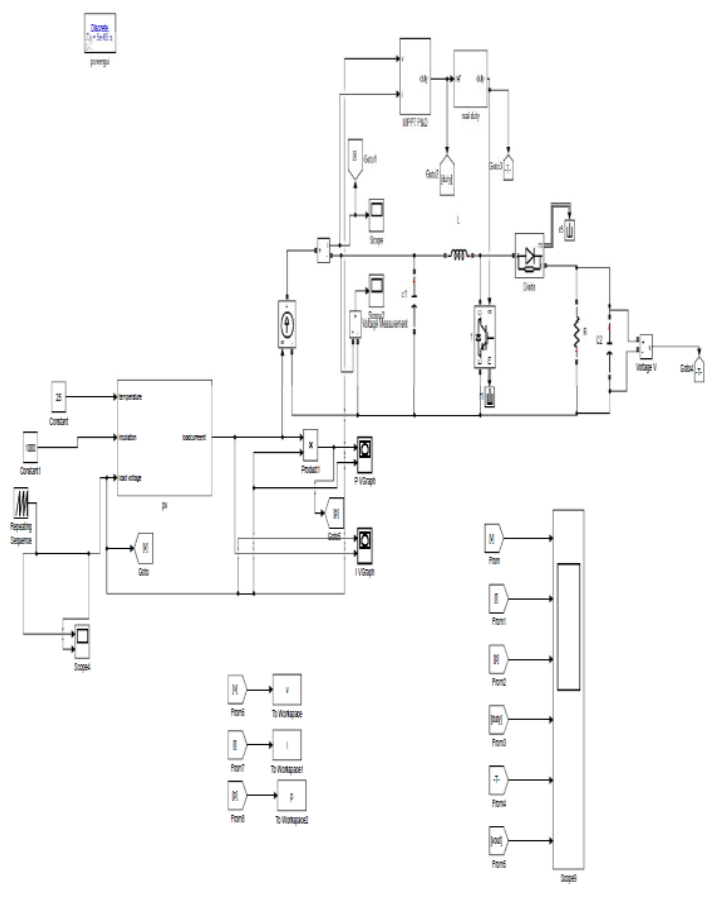

Figure 6: Simulation diagram of MPPT (P\&O) electrical tracking system
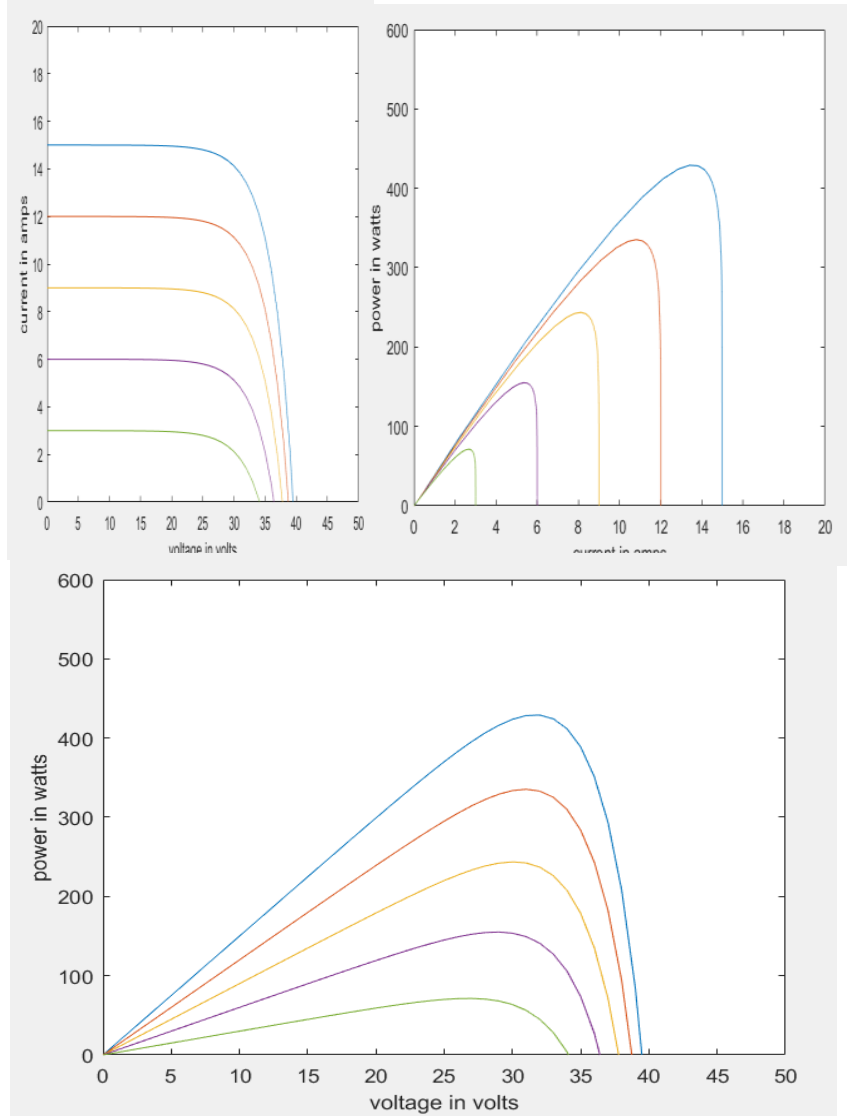

Figure 7: I-V curve, P-V curves-I curves

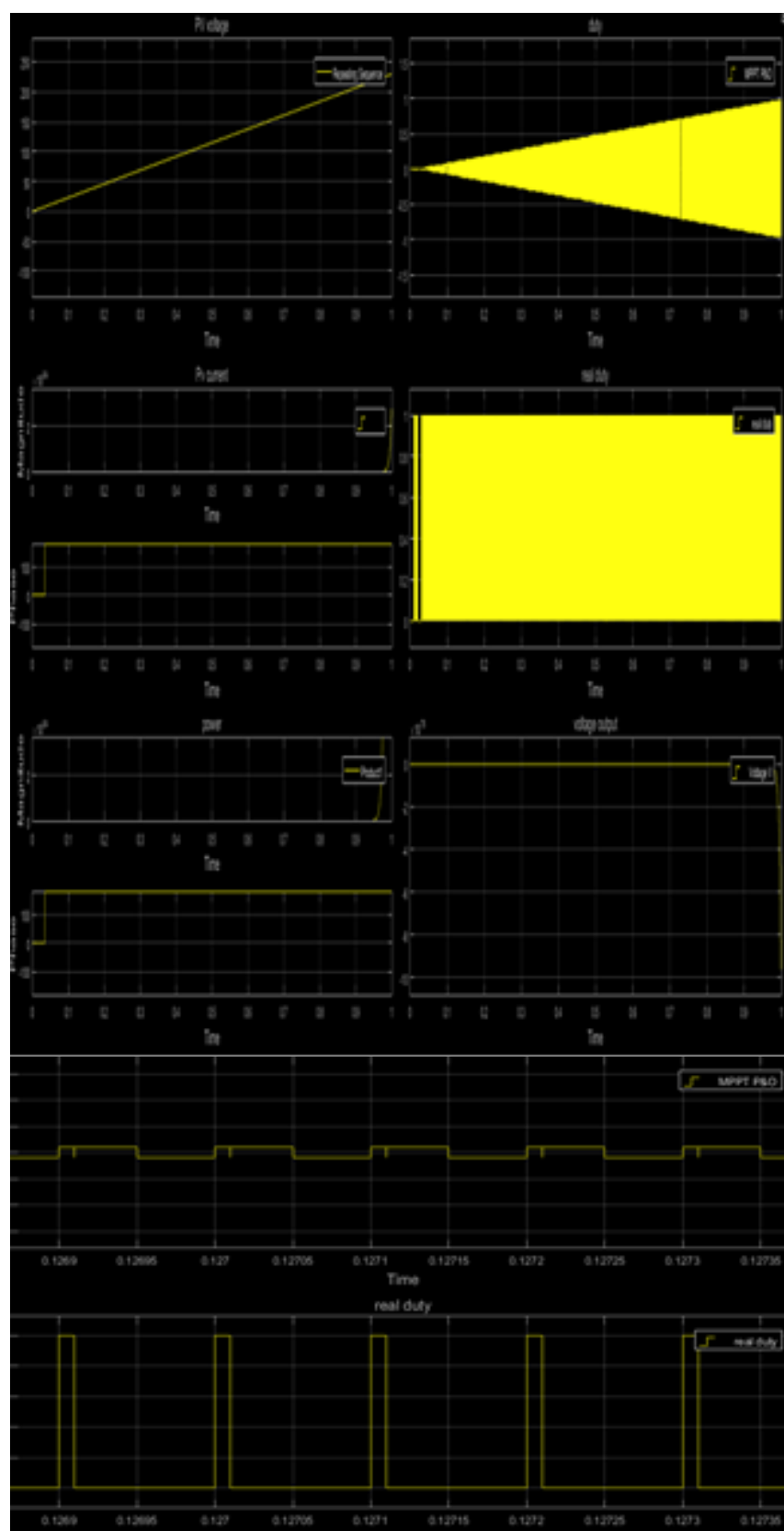

Figure 8: Simulation results of electrical Tracking System

\section{B. Codesys software:}

It is one of the development tool used to program applications which are dealing with controllers in accord with international industrial standard IEC 61131-3. It can be ported to different platforms. German Software Company named 3S-Smart Software Solutions which is located inBavarian town of Kempten introducesCODESYSsoftwarein the Version 1.0 which was released in 1994. CODESYS licenses can be installed for free of charge legally without copy protection. The software tool covers various aspects of evaluated industrial automation technology with one surface.

\section{B.Input/output allocation}

Inputs:

\%IW.1: analog read 1 of LDR1

\%IW2: analog read 1 of LDR2

\%IW3: analog read 1 of LDR3 \%IW4: analog read 1 of LDR4 $\%$ I0.0: start 
\%I0.1: stop

Outputs:

\%QX0.0: DC MOTOR on in direction A $\%$ QX0.1: DC MOTOR on in direction B $\%$ QX0.3: stepper motor on in forward $\%$ QX0.4: stepper motor on in reverse

\section{Simulation results}

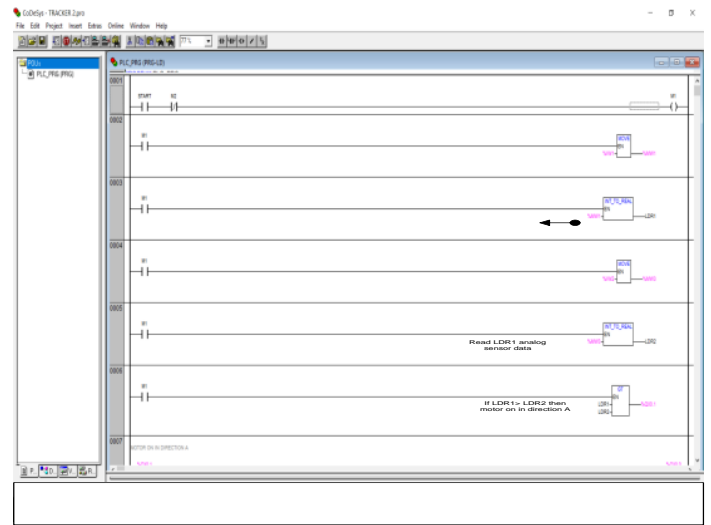

Figure 9: Initial Program in codesys before run simulation

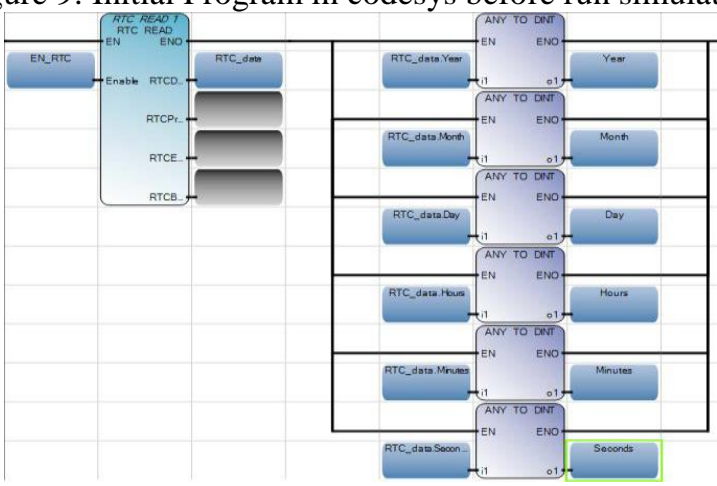

Figure 10 : Real time clock simulation

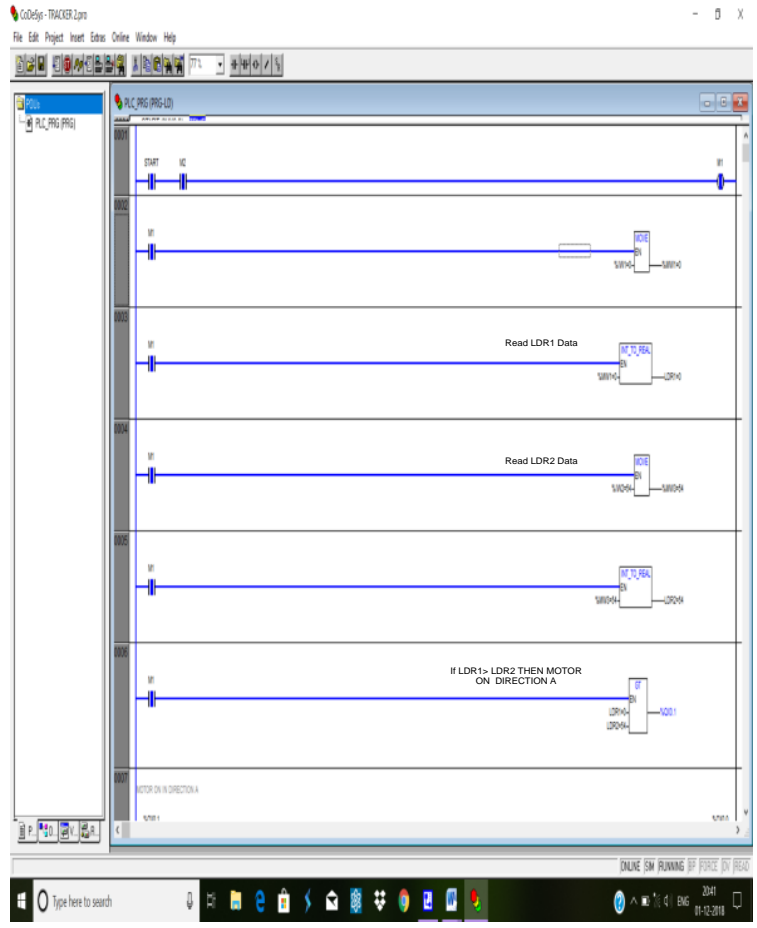

Figure 11: simulation of HL DASTS using simulation software part 1

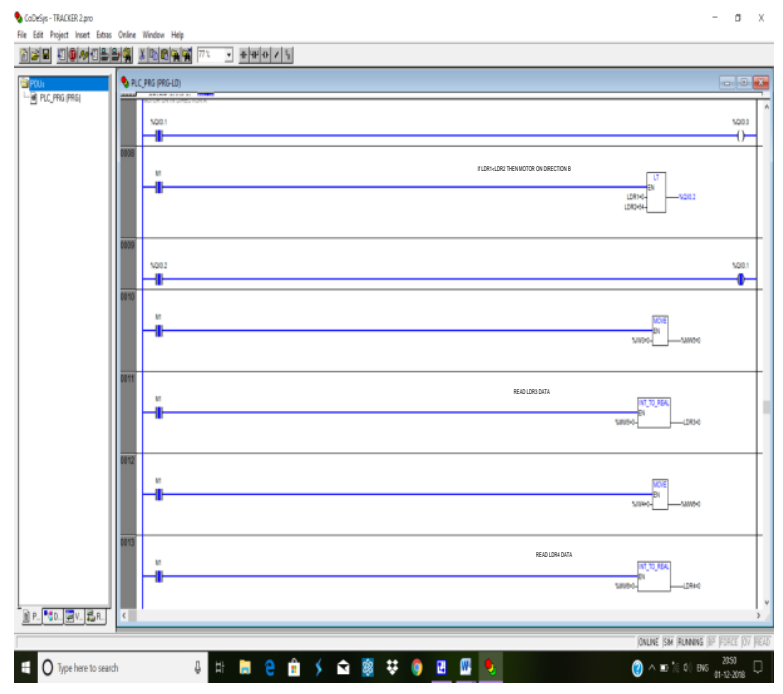

Figure 12: Simulation Result of HL DASTS system Part 2

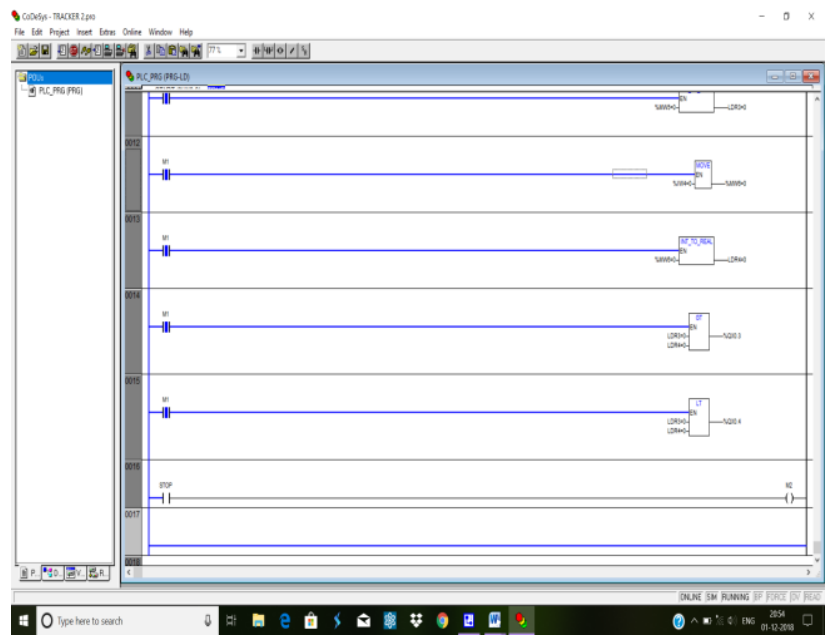

Figure 13: Simulation Result of HL DASTS system Part 3

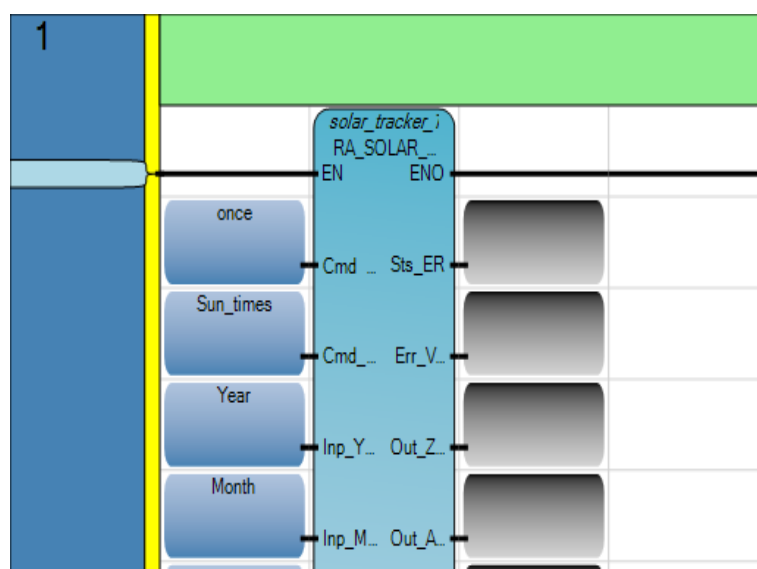

Figure 14: ladder logic with sun position algorithm

\section{CONCLUSIONS}

The Paper focusses on the ladder Programming Implementation in codesys software to perform dual axis solar Tracking system using LDR sensor based closed loop control system and solar positioning algorithm based open loop control system proposed by NREL to find the parameters such as sunrise, sunset, transit time and azimuth, zenith angles for

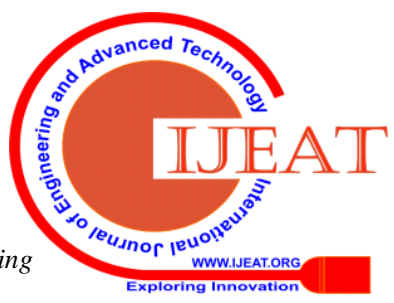


specific location. Maximum power point tracking algorithm based electrical solar tracking in MATLAB platform also performed and respective simulation results incorporated. As per the studies, it is better to choose dual axis hybrid loop solar tracker system for precise and accurate solar output to maximize the efficiency besides, for fixed solar panel MPPT also gives good result but the selection criteria depends on economic, geographical aspects from place to place. Though we execute the SPA by ladder programming other languages such as functional block diagram, structured text may also use, but ladder logic is relatively easier and user friendly. The DASTS with HL control most recommended to residential communities in emerging smart cities where space and efficiency are the key factors. In some applications fixed with electrical tracking systems are involved considering economic factors involved.

\section{REFERENCES}

1. D.B.N Nandi, s e oti, and d.c.Ahiyabukie, Nigerian Journal of Technology (NIJOTECH, volume 33 no.2, april (2014)pages 230-237.

2. YusieRizala r, Sunu Hasta Wibowoa, and Feriyadia, International Conference on Sustainable Energy Engineering and Application, Volume (2012) pages 160-166.

3. Rockwell Manuals, SuneethaRacharla, K. Rajan, International Journal of Sustainable Engineering, volume 10(2017) pages, no. 2, 72-81.

4. Li, Guihua, Runsheng Tang, and Hao Zhang. International Conference on Future Energy, Environment and Materials 16 (2012): 1744-1752.

5. Books: Solar Tracking: High precision solar position algorithms, programs, software and source-code for computing the solar vector, solar coordinates \& sun angles in Microprocessor, PLC, Arduino, PIC and PC-based sun tracking devices or dynamic sun following hardware by gerro j prinloo,robertthomas Dobson(2015)

6. Prof.Dr. Osama Gouda, Assoc. Prof.Dr. GhadaAmer, Dr. Tamer Elkhodary, Proceedings of the 14th International Middle East Power Systems Conference (MEPCON'10), Cairo University, Egypt, December 19-21, 2010, Paper ID 129.

7. Lakhvir Singh, Jujhar Singh, American Journal of Computer Science and Engineering Survey, ISSN 2349 - 7238

8. Mousazadeh, H., Keyhani, A., Javadi, A., Mobli, H., Abrinia, K., Sharifi, A Renewable and Sustainable Energy Reviews, Jan. 2009. Vol 13, issue 8, pp.1800-1818.

9. Mwithiga G, Kigo SN, Journal of Food Engineering 2006, vol 74, issue 2, pp. 247-252.

10. Bingol O, Altintas AO, Journal of Engineering Sciences 2006, vol. 12, pp. 243-248

11. KamleshYadava, O.S. Sastrya, R. Wandhare, N. Sheth,M. Kumar,B. Bora, Rashmi Singh, Renu A. Kum,Solar Energy 119 (2015) 195-202.

12. G. Ganesh, G Vijay Kumar, A.R.VijayBabu, G.Srinivasa Rao, Y.R.Tagore, Performance Analysis and MPPT Control of a Standalone Hybrid Power Generation System, Journal of Electrical Engineering, ISSN: 1582-4594, Volume 15, Edition: 1, pp. 334-343,2015.

13. L. M. Elobaid, A. K. Abdelsalam, and E. E. Zakzouk, "Artificial neural network based maximum power point tracking technique for PV systems," in Proceedings of the 38th Annual Conference on IEEE Industrial Electronics Society (IECON '12), pp. 937-942, Montreal, Canada, October 2012.

14. Abiramasundari, Mrs. S, Gayathiri D, Mehala K, Sivaranjini s, and Kousalya R. "Design of smart toll cash collection using nfc reader." international journal of communication and computer technologies 7.supplement 1 (2019), 19-23. Print. Doi:10.31838/ijccts/07.sp01.04

15. MnBorhan,'Design Of The High Speed And Reliable Source Coupled Logic Multiplexer",Journal of VLSI Circuits And Systems 1 (01), 18-22,2019

16. Sukumar, Durga, JayachandranathJithendranath, and SumanSaranu. "Three-level inverter-fed induction motor drive performance improvement with neuro-fuzzy space vector modulation." Electric Power Components and Systems 42.15 (2014): 1633-1646.

17. Yadlapalli, Ravindranath Tagore, and AnuradhaKotapati. "A fast-response sliding-mode controller for quadratic buck converter." International Journal of Power Electronics 6.2 (2014): 103-130. 